\title{
Abdominal Ganglion Neuro Endocrin Neoplasm: A Case Report
}

\author{
Sania Abdul Rehman ${ }^{1 *}$, Hajra Sarwar ${ }^{2}$, Muhammad Afzal ${ }^{3}$
}

Lahore School of Nursing, The University of Lahore, Pakistan

DOI: $10.36348 /$ sjnhc.2019.v02i12.009

| Received: 05.12.2019 | Accepted: 14.12.2019 | Published: 28.12.2019

*Corresponding author: Sania Abdul Rehman

\section{Abstract}

Introduction: The neuroendocrine neoplasms (NENs) are comparatively infrequent and mixed tumors which comprise $2 \%$ of all malignancies, with an incidence of $<200,000$ population. Gastrointestinal neuroendocrine carcinomas are diagnosed rarely. While gastric carcinoma is third principal reason of death in men and fifth in women especially in Eastern Asia. Case Report: We, herein, present a patient of 30 years and male who was presented to OPD having complaints of diarrhea, hot flushes, dizziness, dyspepsia and anorexia. A Core Biopsy of left par aortic region was made. The findings showed that patient was suffering from neuroendocrine neoplasm/paraganglioma. Initially, lesions can be managed by endoscopic excision of accessible tumors and endoscopic monitor. Discussion: Abdominal neuroendocrine carcinomas are also rare and accounting for less than $10 \%$ of gastric neoplasms and such rareness has made it tough to accurately recognize their organic nature and to identify ideal treatment choices. Conclusion: As we described a patient of intermittent gastric neuroendocrine carcinoma, a well-managed description of these cancers/tumors should be discussed globally and historically. Diagnostics and therapeutic managements must be evaluating in large clinical studies. Keywords: Neuroendocrine Neoplasm, Gastric Neuroendocrine.

Copyright @ 2019: This is an open-access article distributed under the terms of the Creative Commons Attribution license which permits unrestricted use, distribution, and reproduction in any medium for non-commercial use (NonCommercial, or CC-BY-NC) provided the original author and source are credited.

\section{INTRODUCTION}

Gastric neuroendocrine tumors emerge from enterochromaffin like cells present in the mucosa of stomach. With reference to histology, serology, and endoscopy findings, they can be distributed into types I, II, and III with aggressiveness degree varying[1]. Gastrointestinal neuroendocrine carcinomas are diagnosed rarely [2]. A survey from the US's Surveillance Epidemiology and End Results (SEER) Register depicts that neuroendocrine carcinomas of small intestine have been increased 300\%-500\% in last 35 years. Same numbers are being seen in Sweden and Norway. The etiology is unknown[3]. While gastric carcinoma is third leading cause of death in men and fifth in women especially in Eastern Asia [4].

The neuroendocrine neoplasms are uncommon and mixed tumors which include $2 \%$ of all malignancies, with a prevalence of $<200,000$ population [5].

\section{CASE REPORT}

A patient of 30 years and male was presented to OPD. He was having the complaints of diarrhea, hot flushes, dizziness, dyspepsia and anorexia. These symptoms started a year before admission. The blood pressure was high 145/100. He was evaluated 3 weeks before presentation. No history of family was reported regarding any disease. Heart malfunctioning was diagnosed and level of hemoglobin was $10 \mathrm{~g} / \mathrm{dl}$. History of hypertension was reported as well as extra gastric hormonal syndromes discussed above i.e. diarrhea, flushes. Endoscopic examination of upper abdominal part revealed localized ulcerative lesion. A Core Biopsy of left par aortic region was made. The findings showed that patient was suffering from neuroendocrine neoplasm/paraganglioma. The tumor cells showed hyper chromatic nuclei along with intense mitosis. There was also nymph node involvement (Lymphatic invasion). On immune histochemical staining, the tumor was positive for synaptophysin and negative for S-100 protein. Due to these findings and long follow up, it was concluded that this is neuroendocrine carcinoma according to WHO classification [6]. Moreover, tumor was uniform in shape, having solid nest like structure.

\section{DISCUSSION}

Enterochromaffin like cells are no peptide secreting endocrine cells of stomach mucosa. It includes $30 \%$ of endocrine cells. Histamines are released in response to gastrin production by $\mathrm{G}$ cells. Long term release is the reason of hyperplasia [7]. 
WHO defines and differentiated neuroendocrine tumor made of cells with structures similar to those which are normal endocrine gut cells. They express universal indicators of neuroendocrine differentiation and hormones according to site having mild to moderate nuclear atypical and low number of mitosis [8].

Gastric neuroendocrine neoplasm (NENs) includes a cluster of tumors with a series of histopathological varieties extending clearly from benign to extremely malignant tumors. Recent changes have been made to the concept of this illness and its investigative condition. Stomach gastric neuroendocrine neoplasms are characterized as neoplasms with neuroendocrine variation in 2010 WHO criteria, including neuroendocrine tumors and carcinomas. Synonyms for gastric neuroendocrine tumors include carcinoids which are well differentiated while carcinomas include poorly differentiated carcinoma [6].

NENs are classified into endocrine tumor and carcinoid tumors which may be small cell type or large type according to Japanese gastric carcinoma classification [9].

The incidence of gastric neuroendocrine neoplasms has increased recently. Those tumors assume to be generally uncommon which is account for less than $1 \%$ of all gastric tumors [10]. Most of these tumors are generally neuroendocrine tumors whose course is not life undermining. Over hundred years have been passed since the term 'carcinoid' proposed by Oberndorfer in 1907 [11].

Gilligan concluded a treatment for carcinoid tumors in this context including the above subtypes of tumors. Initially, lesions can be treated by endoscopic excision of nearby tumors and endoscopic monitoring. If tumors still reappear, it is possible to use antrectomy and local excision to eliminate the gastrin source. Less invasive therapeutic options have recently been reported such as endoscopic tumor resection for small neuroendocrine tumors [12].

Abdominal neuroendocrine carcinomas are also rare and accounting for less than $10 \%$ of gastric neoplasms and this rareness has made it difficult accurately to know their genetic nature and to identify ideal treatment options [13].

Our case's neuroendocrine carcinoma was a challenging diagnosis and immunohistochemistry played a key part. Microscopically, the tumor of the patient was uniform in shape and set to form solid nests with hematoxyl and eosin staining medium -sized round to cuboid-to cuboid cancer cells. Usually, the tumors are positive for synaptophysin \& neuronal specific enolase. But less than often, they are positive for chromogranin a staining. Intense lymph invasion was seen in many nodes which suggested it as a malignant tumor of high grade. For many of them chemotherapy and surgery should be done.

\section{CONCLUSION}

Malignant paraganglioma are rare carcinomas that are why its diagnosis and treatment is a difficult task. Team work implant can facilitate the proper diagnosis and therapy. As we described a case of infrequent gastric neuroendocrine carcinoma, a wellmanaged description of these cancers/tumors should be discussed globally and historically. Diagnostics and therapeutic managements must be evaluating in large clinical studies.

\section{ACKNOWLEDGMENT}

The author declared that there is no financial support received in this case study.

\section{REFERENCES}

1. Modlin, I. M., Lye, K. D., \& Kidd, M. (2003). Carcinoid tumors of the stomach. Surgical oncology, 12(2), 153-172.

2. Chin, J. L., \& O'Toole, D. (2017). Diagnosis and management of upper gastrointestinal neuroendocrine tumors. Clinical endoscopy, 50(6), 520.

3. Xavier, S., Rosa, B., \& Cotter, J. (2016). Small bowel neuroendocrine tumors: from pathophysiology to clinical approach. World journal of gastrointestinal pathophysiology, 7(1), 117

4. Kubota, K., Okada, A., Kuroda, J., Yoshida, M., Ohta, K., Adachi, M., ... \& Kitajima, M. (2011). Neuroendocrine carcinoma of the stomach: a case study. Case reports in medicine, 2011.

5. Shimoyama, S., Fujishiro, M., \& Takazawa, Y. (2010). Successful type-oriented endoscopic resection for gastric carcinoid tumors: A case report.World journal of gastrointestinal endoscopy, 2(12), 408.

6. Bosman, F. T., Carneiro, F., Hruban, R. H., \& Theise, N. D. (2010). WHO classification of tumours of the digestive system (No. Ed. 4). World Health Organization.

7. Oronsky, B., Ma, P. C., Morgensztern, D., \& Carter, C. A. (2017). Nothing but NET: a review of neuroendocrine tumors and carcinomas. Neoplasia, 19(12), 991-1002.

8. Kwon, D. H., Nakakura, E. K., Bergsland, E. K., \& Sun-Chuan, D. (2017). Gastric neuroendocrine tumors: management and challenges. Gastrointestinal Cancer: Targets and Therapy, 7, 31 .

9. Japanese Gastric Cancer Association. (2011). Japanese classification of gastric carcinoma: 3rd English edition. Gastric cancer, 14(2), 101-112.

10. Chiba, N., Suwa, T., Hori, M., Sakuma, M., \& Kitajima, M. (2004). Advanced gastric endocrine cell carcinoma with distant lymph node metastasis: 
a case report and clinicopathological characteristics of the disease. Gastric Cancer, 7(2), 122-127.

11. Larsson, L. I. (2000). Developmental biology of gastrin and somatostatin cells in the antropyloric mucosa of the stomach. Microscopy research and technique, 48(5), 272-281.

12. Kim, E. H., \& Park, C. H. (2017). Screening endoscopy for gastric cancer: time for quality control. Translational gastroenterology and hepatology, 2.

13. Scherübl, H., Jensen, R. T., Cadiot, G., Stölzel, U., \& Klöppel, G. (2010). Neuroendocrine tumors of the small bowels are on the rise: Early aspects and management. World journal of gastrointestinal endoscopy, 2(10), 325. 